\title{
Design of high precision current signal source on DDS
}

\author{
Han Ya Kun ${ }^{1, a}$, Suo Xue Song ${ }^{\star, 1, b}$ \\ ${ }^{1}$ Agricultural University of Hebei, Baoding, Hebei Province ,China \\ aemail: 1063871384@qq.com bemail: 13903120861@163.com \\ * Corresponding Author, Suo Xuesong
}

\begin{abstract}
Keywords: electrical net detect; AT89C51 single chip computer; signal conditioning; signal source;AD9851; complementary push-pull amplifier circuit.
\end{abstract}

\begin{abstract}
To provide a amplitude, frequency programmable current signal with high precision for fault detection of $10 \mathrm{kv}$ rural power line, this paper presented a high precision current signal source using DDS (Direct Digital Frequency Synthesis) technology. The system includes two parts which are signal generator part and control part. The system uses DDS chip AD9851 as the main signal generator, AT89C51 as the main controller, Use the keyboard to select the frequency, phase and amplitude, USE LED display the frequency and amplitude. As the experimental result shows, the system has a stable performance, and the output signal, generated by the system, has advantages of high accuracy and good adjustability for fault detection on $10 \mathrm{kv}$ transmission line.
\end{abstract}

\section{Introduction}

The level of rural power grid has been greatly improved compared with the previous through modification inn the past few years. But the failure rate of the rural $10 \mathrm{kV}$ distribution network is still high. This is because of openness and relatively large volatility of rural power grid ${ }^{[1]}$. Therefore, the fault detection and repair for rural power grid is still very important. To provide an amplitude and frequency programmable current signal with high precision for fault detection of $10 \mathrm{kv}$ rural power line. It is necessary to provide an adjustable current signal source. However, the traditional signal sources are made of integrated chip MAX038 or ICL8038, which connecting capacitive or resistive by self-oscillation ${ }^{[2]}$. Due to environmental factors greater impact on the external device, Traditional signal source output signal is less stable, while the high-frequency signal output can be limited ${ }^{[3,4]}$. It is not well adapted to the rural power grid fault detection. Therefore, the design of a simple structure, the influence of external factors small, flexible working, high precision current signal source, to meet the special nature of rural power grid fault detection, has important practical significance.

\section{System components}

The current signal source includes two parts which are signal generator part and control part., the structure shown in Figure 1. The control module is the core of the whole system, consisting of the AT89C51 microcontroller, $4 \times 2$ keyboard, LED. AT89C51 microcontroller through keyboard input selected sine current signal amplitude、 frequency ,use LED display input data to ensure the accuracy of the data. Then MCU sends the initial input parameter to the signal generation module. Signal generation module (AD9851) to produce the corresponding sinusoidal current signal based microcontroller instruction, the signal flow through the low pass filter (LPF)、 the high pass filter (HPF) and signal conditioning circuit. The A/D chip collect output sine signal and put collected digital signal to AT89C51 microcontroller. After calculating AT89C51 microcontroller compared with the user set value to ensure that the output sine current signal is standard. 


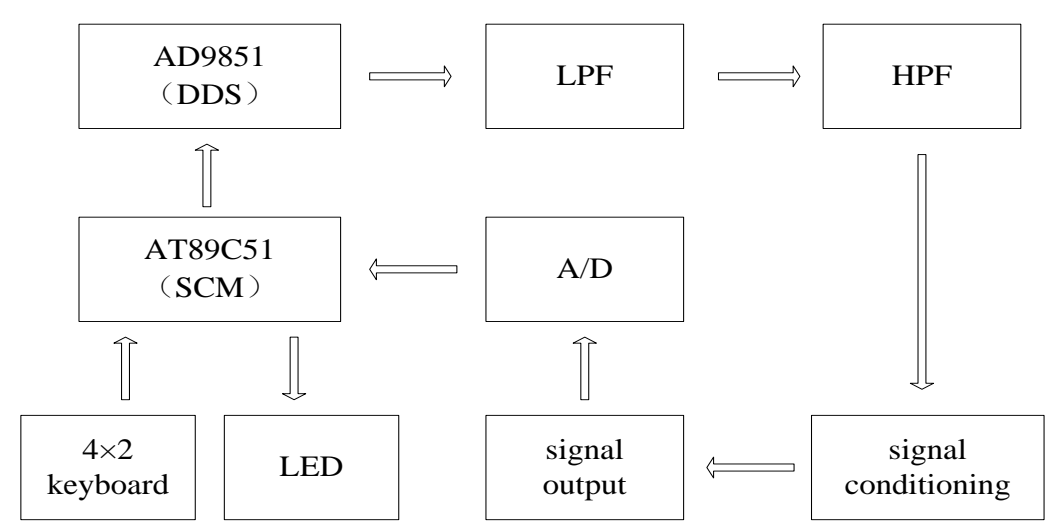

Figure 1 The principle diagram of the signal source

\section{System hardware design}

Microcontroller selection. Signal source using AT89C51 as the control center. This microcomputer has $4 \mathrm{k}$ byte Flash memory, 128 bytes in the internal RAM, 32 programmable I/O lines, two 16 bit timer/counter, a five vector two-level interrupt structure, a full duplex serial communication port, the on-chip oscillator clock and clock circuitry ${ }^{[5]}$. AT89C51 supports two programming language Assembler and C language. While the programming is simple, it is easy to realize control of the AD9851 programming; Due to small size and low price, it is easy to implement miniaturization of the system. So using AT89C51 microcontroller as the control center of the system sends control words to AD9851.

Design of AD9851 chip interface circuit . AD9851 made in ADI company that use advanced DDS technology is highly integrated DDS frequency synthesize. Its interior comprises a programmable DDS systems, high performance DAC and high-speed comparators, which can achieve all-digital programming control of frequency synthesis and clock generation ${ }^{[6,7]}$. AD9851 interface function control is simple, it can use 8-bit parallel port or serial port directly to the input control data as frequency, amplitude.

When outputting adjustable frequency sine current signal, microcomputer load frequency control word to AD9851 using serial way. AD9851 output corresponding sine current signal according to the frequency of the internal registers, AD9851's W_CLK and FQ_UD connected to the microcontroller $\mathrm{P} 1 \wedge 0$ and $\mathrm{P} 1 \wedge 1$.

When AD9851 output sine current signals, whose output of the sine current signal amplitude can be set by an external resistor $R_{S E T}{ }^{[8]}$, the calculation formula of current

$$
I_{S E T}=\frac{(32 \times 1.248)}{R_{S E T}}
$$

So using digital potentiometer AD8400 changes the input resistor $R_{S E T}$ to obtain different amplitude of the current signals. To prevent short circuits, it needs the connection resistance between the AD8400's output port B1 and $R_{S E T}$ with a value of $3.9 \mathrm{~K} \Omega$. AD8400 control word has 10 bits, whose last eight bits control resistance, so there are $2^{8}=256$ different resistance values. AD8400 choose standard resistance value of $100 \mathrm{~K} \Omega$ resistor, $\mathrm{RAB}=100 \mathrm{k} \Omega$. So the $R_{S E T}$ resistance range $3.9 \mathrm{k} \Omega$ to $103.9 \mathrm{k} \Omega, 3.9 \mathrm{k} \Omega \leqslant R_{S E T} \leqslant 103.9 \mathrm{k} \Omega$. Into formula (1), calculating the output current ranges $0.3865 \mathrm{~mA}$ to $10.12 \mathrm{~mA}, 0.3865 \mathrm{~mA} \leqslant \mathrm{I}_{\mathrm{SET}} \leqslant 10.12 \mathrm{~mA}$.

Design of the filter circuit. In order to eliminate the spurious interference of the output signal to get higher precision of sine current signals, it must be design filter for the signal source. Since the signal source output signal frequency range for $3 \mathrm{kHz}-10 \mathrm{kHz}$. The amplifier has the characteristics of high input impedance and low output impedance. After considering the frequency response, ripple, bandwidth, and other parameters, the system uses a Second-order Butterworth low-pass filter and a high pass filter consist of two levels of RC filter circuit and in phase proportion amplifier circuit $^{[9]}$.Schematic diagram shown in Figure 2, by the formula 


$$
f=\frac{1}{2 \pi R C}
$$

There are capacitance and resistance parameters. The low-pass cutoff frequency is $10.6 \mathrm{kHz}$, and the high-pass cutoff frequency is $2.84 \mathrm{kHz}$ by calculating, meeting the filtering requirements.

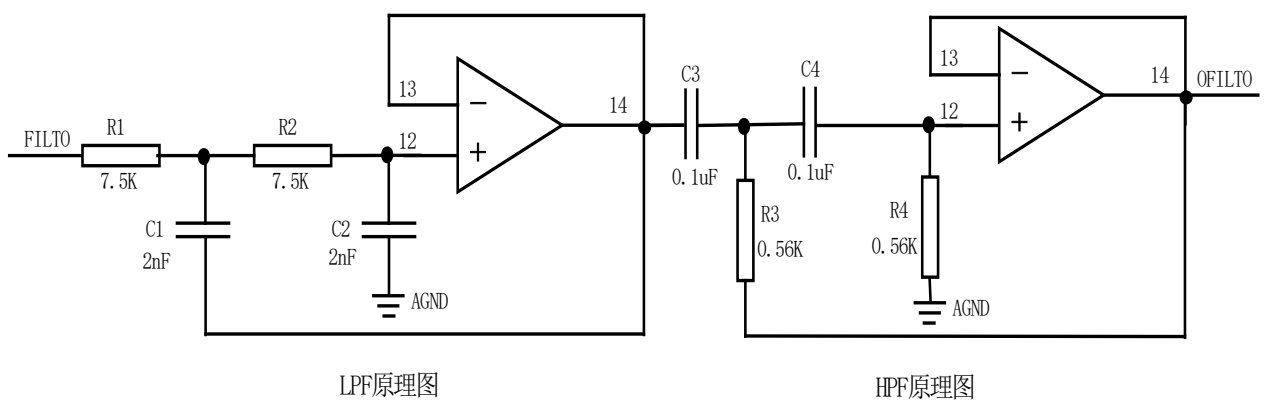

Figure 2 The diagram of filter principle

Design of signal conditioning circuit. In order to improve the load-bearing ability and the power output ability of the signal source, it needs amplify the sine current signals that AD9851 output filtered through filter. Signal conditioning block diagram shown in Figure 3 . The sine current signal is connected to signal input terminal of the multiplier, the output signal of the feedback circuit connected to the other signal input terminal of the multiplier, to achieve stable current through feedback control. When the output current is bigger, the feedback circuit of the output current is reduced, the multiplier output is less, so as to reduce the output current to normal. On the other hand, the adjustment is set up. Even if the load changes, the current value of the output is always keep constant.

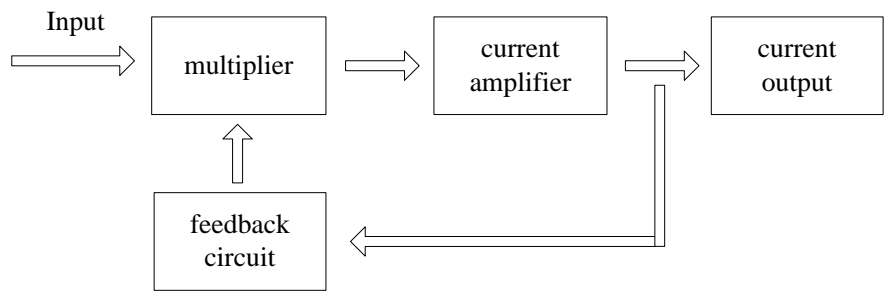

Figure 3 The block diagram of signal conditioning

Because most integrated operational amplitier maximum output current is only tens of milliamps, it can not meet the requirement of the large load current. The traditional method uses a complementary push-pull amplifier circuit consist of the two different polarities transistor, But this amplifier exists crossover distortion. Therefore, this system has been improved to such circuit, eliminated the distortion of the complementary push-pull amplifier circuit. Circuit is shown in Figure 4. The four diodes in series, by adjusting potentiometer $\mathrm{W}$, the circuit is turned on, thereby eliminating crossover distortion.

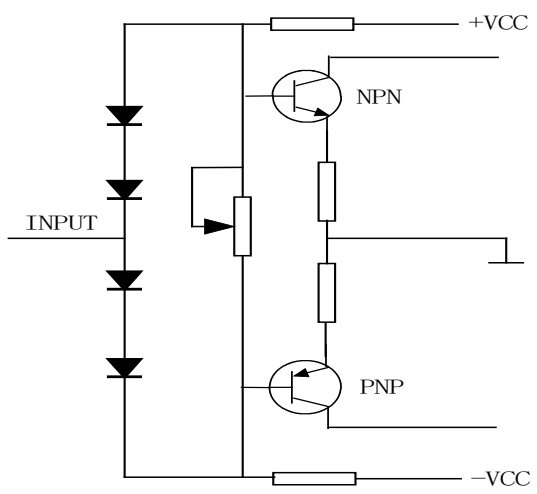

Figure 4 Eliminate the distortion of the amplifying circuit 
Signal output and acquisition. To ensure high precision of sine signal waveform, using a A/D sampling chip to collect current signal in current output. A/D acquisition chooses ADC0832 chip, that is an 8-bit resolution, dual-channel A / D converter chip. It is characterized by small size, compatibility, etc ${ }^{[10,11]}$. AD0832 will send the acquiring digital signal to the microcontroller. By calculating the microcontroller adjusts the output signal to ensure that the output sine current signal is standard.

\section{Software process system design.}

System software part modular written with C language, is mainly composed of initialization procedures, key interrupt program, display program. The total design flow chart and key interrupt program flow chart is shown in Figure 5.

\section{Experiment and result analysis.}

According to the above design of hardware circuit, using Proteus simulates signal conditioning circuit. Testing loads with pure resistance or inductance circuit, and using the digital oscilloscope display the output sine signal. The simulation results are shown in figure 6 . In the picture the small amplitude curve is the basic input signal and the large amplitude curve is the output signal after the signal conditioning.

The experimental results show that there changes in load almost have no effect on the frequency and amplitude of the sine signal. The frequency and amplitude of the signal output are stability, meeting the design requirements.

\section{Summary}

The current signal source is an important component of the grid detection device. Highly accurate sine current signal to ensure power system reliability of power supply plays an important role. In this paper, based on DDS technology, using AT89C51 as controller achieves the design of precision current variable frequency signal source. The paper creatively use complementary push-pull amplifier circuit which has the elimination of crossover distortion function and feedback regulation to amplify the signal. The experimental results show that the signal source provides accurate stable sinusoidal signal for fault detection on rural $10 \mathrm{kV}$ distribution network, improve the reliability of power supply in rural power grid, reduce the failure rate due to signal error generated, let the grid fault detection more accurate. 

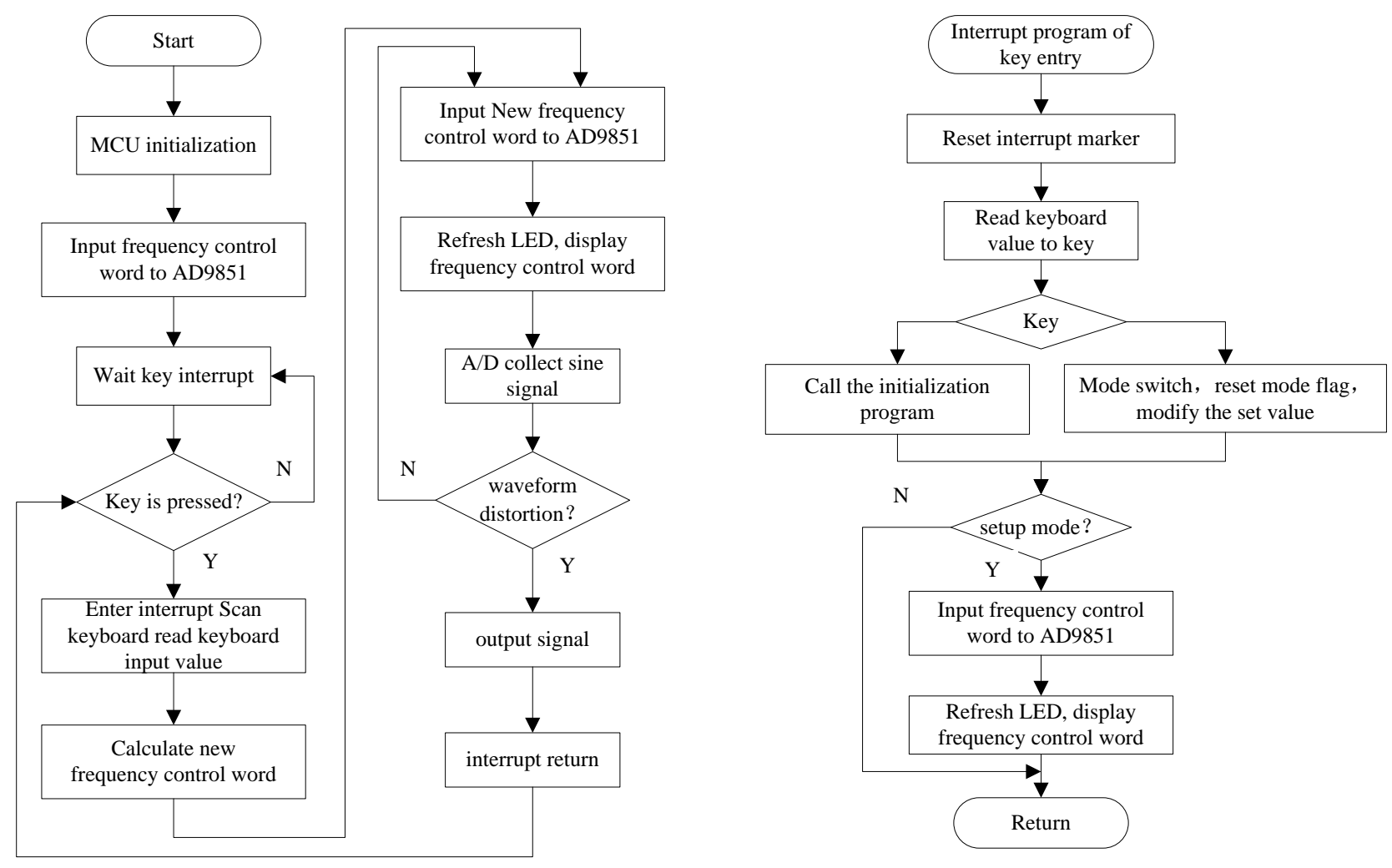

Figure 5 The flow chart of software system and key interrupt system

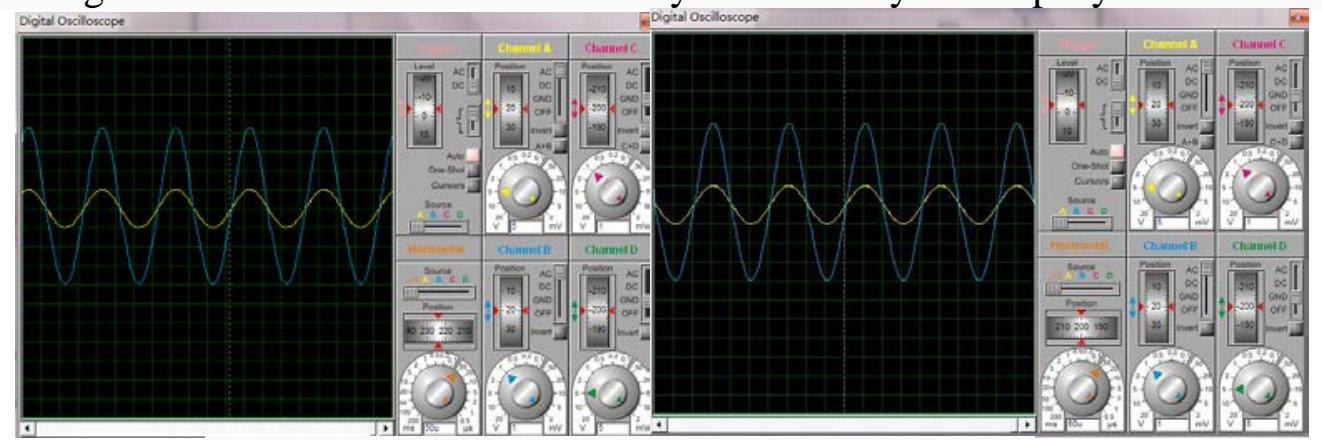

Figure 6 The picture of signal disposal simulation

\section{Acknowledgment}

Program of Study Abroad for Young Teachers by Agricultural University of Hebei; Young Academic Leaders of Hebei Agricultural University; Polytechnic Fund of Agricultural University of Hebei ( ZD201402)

\section{References}

[1] Li li, Zhang Dao jun, Wan mei and Cheng sheng. The analysis of present rural network situation and the rural electric reform problems for our country[J]. Technology Guide. 2012（35): 229-230

[2] Shi Yan dong, Chen Guo rui and Ning Fei. Design and Application of Three-phase Sinusoidal Signal Generator Based on AD9834 [J]. Machinery \& Electronics, 2010 (10) : 13-17.

[3] Gu Zhao yu, Wang ping , Fu qi xiang. Design and implementation of a signal source based on DDS [J]. Modern Electronic Technique, 2015(5):23-25

[4] V.F.Kroupa Spurious signal in direct digital frequency synthesizer due to the phase truneation.2000 IEEE Transactions on ultrasonic ,And frequency control:1166-1172 
[5] Zhang Lan Hong, Zou Hua. Single chip microcomputer principle and application [M]. Beijing: Mechanical industry press, 2012

[6] Xu Xiao lin, Liu Si Xin. The design and implementation of stepping frequency source based on DDS chip AD9851 [J]. POPULAR SCIENCE \& TECHNOLOGY. 2009(05):62-63

[7] Analog Devices Inc..CMOS.125 MHz computer DDS synthesizer AD9851[M].[S.1.]: Analog Devices Inc.,1999

[8] Ying Zhao Long, Cai Xiang, Wang Cheng Cheng. Design and implementation three-phase AC source based on AD9850 [J]. ELECTRONIC MEASUREMENT TECHNOLOGY. 2011:8-11

[9] Zeng Zhi hui, Wen Zhi peng, Liu Cheng yuan. A new real-time detection method for power system harmonics based on algebraic transformation of sine cosine. Journal of Electric Power Science and Technology. 2011(3):93-95

[10] Li Ru Shan,Sun Yan Ping Zhang Er wen. High Precision Electronic Detection of Drug Weight [J]. MECHANICAL AND ELECTRONIC INFORMATION. 2013(14):26-29

[11] Wang Yang, Wu Qing Hui and Pang Yan Wei. The application of ADC0832 in detection of the vacuum degree data [J]. Electronic Design Engineering.2013(9):179-180 